\title{
eficacia resistente de pilares de hormigón armado de baja calidad
reforzados por dos procedimientos diferentes
}

J. L. RAMIREZ ORTIZ, Prof. Dr. Ingeniero Industrial J. M. BARCENA DIAZ, Ingeniero Industrial

$831-32$

s i n o p i s La investigación trata sobre la posibilidad de refuerzo en pisos aislados de pilares de hormigón armado de baja calidad, por dos métodos usuales: el recrecido de la sección transversal con hormigón y el adosado de angulares de acero a las aristas del pilar.

La experimentación ha consistido en la fabricación, refuerzo y ensayo de dieciocho pilares de hormigón armado de baja calidad. A una cierta altura sobre sus bases se dispuso un ensanchamiento para representar la presencia de un forjado plano.

De los ensayos de compresión se han sacado datos y conclusiones referentes a la eficacia de los dos procedimietos de refuerzo, distribución de esfuerzos entre pilar original y refuerzo, deformaciones, agrietamientos y forma de rotura.

\section{INTRODUCCION}

La justificación del presente trabajo ha sido el conocer experimentalmente el comportamiento a compresión simple de pilares de hormigón armado de calidad inferior a la exigida por el proyecto, reforzados en pisos aislados por dos procedimientos: el recrecido de su sección transversal por medio de un determinado espesor de hormigón armado y el acoplado de angulares de acero laminado a las cuatro aristas del pilar con unión entre ellos a base de presillas.

En particular se ha estudiado dicho comportamiento resistente, como hemos dicho, en el caso de reforzar pilares aisladamente, sin establecer continuidad del refuerzo hacia arriba y hacia abajo en toda la línea del pilar, pues de esta última forma la capacidad resistente del refuerzo se aprovecha en su mayor parte, pero con coste alto.

El caso más problemático es efectivamente el reforzar pilares aisladamente, pues el refuerzo, al entrar en carga, se apoya en mayor o menor grado en la resistencia al corte del forjado superior e inferior en su unión con el pilar, ya que la sección transversal de dicho refuerzo queda por fuera de la sección del pilar superior y muchas veces también de la del inferior. Con refuerzo en hormigón ar- mado habrá una transmisión por rozamiento $y$ adherencia entre el pilar original y el hormigón de refuerzo y será menos problema el corte del forjado; en el caso de refuerzo metálico, por el contrario, no se puede contar con rozamiento y adherencia entre angulares de refuerzo y pilar original y será crítico el corte del forjado.

\section{DIMENSIONES DE PILARES Y REFUERZOS}

La sección del pilar original de proyecto ha sido de $25 \times 25 \mathrm{~cm}$, con una armadura de 4 redondos de acero liso, de $10 \mathrm{~mm}$ de diámetro, y estribos, de $4,5 \mathrm{~mm}$ de diámetro, separados $15 \mathrm{~cm}$, también en acero liso. Como puede observarse se ha utilizado una armadura muy reducida, inferior incluso a la minima de normas, para que fuera la calidad del hormigón la que condicionara principalmente la resistencia a compresión del pilar.

\footnotetext{
- Los trabajos de investigación se han realizado merced a la Ayuda a la Investigación concedida por el Ministerio de Educación y Ciencia a sus autores, en el marco de la Cátedra de Estructuras de la Escuela Superior de Ingenieros Industriales, de la que J. L. Ramírez Ortiz es titular.

Los ensayos se han realizado en los Laboratorios de la Cátedra de Estructuras y en los Laboratorios de Ensayos e Investigación Industrial «L. J. Torrontegui», anexos a dicha Escuela.
} 


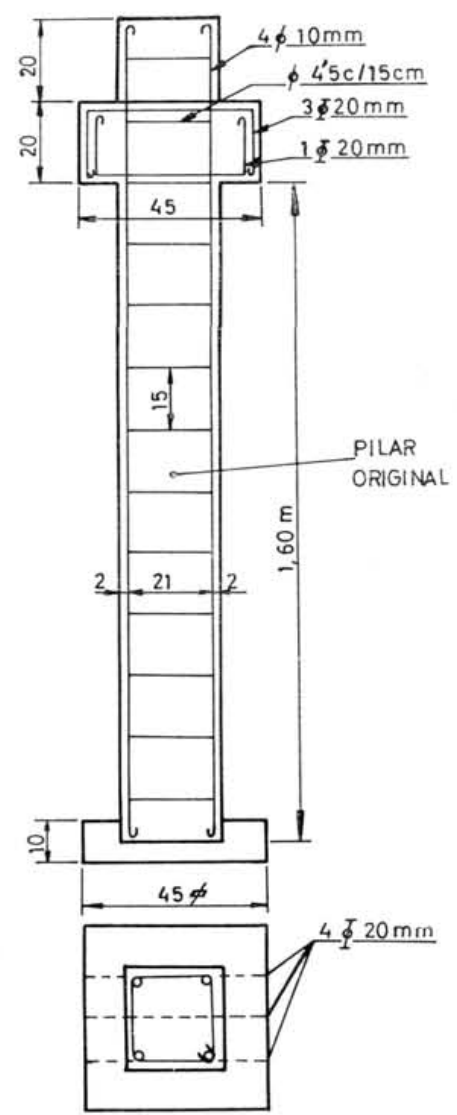

Fig. 1
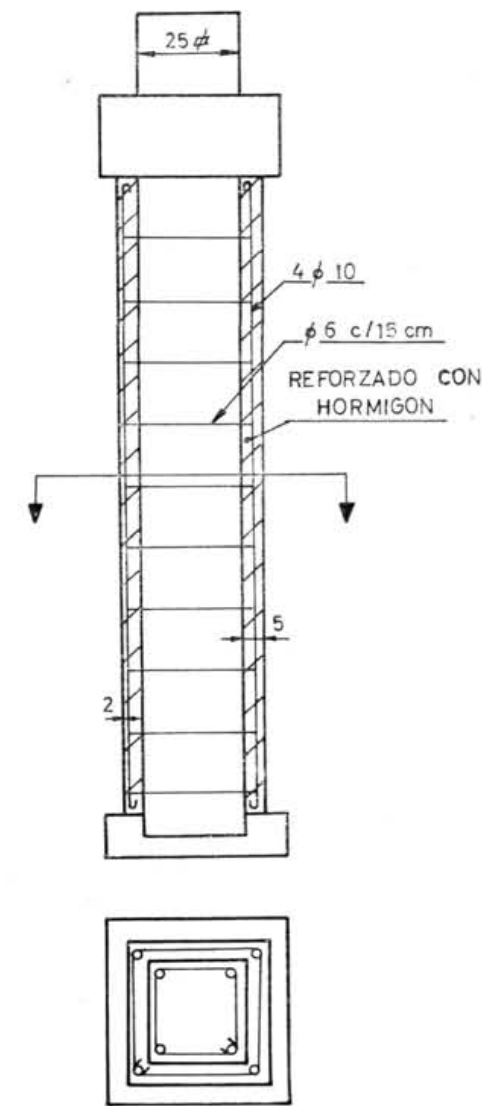
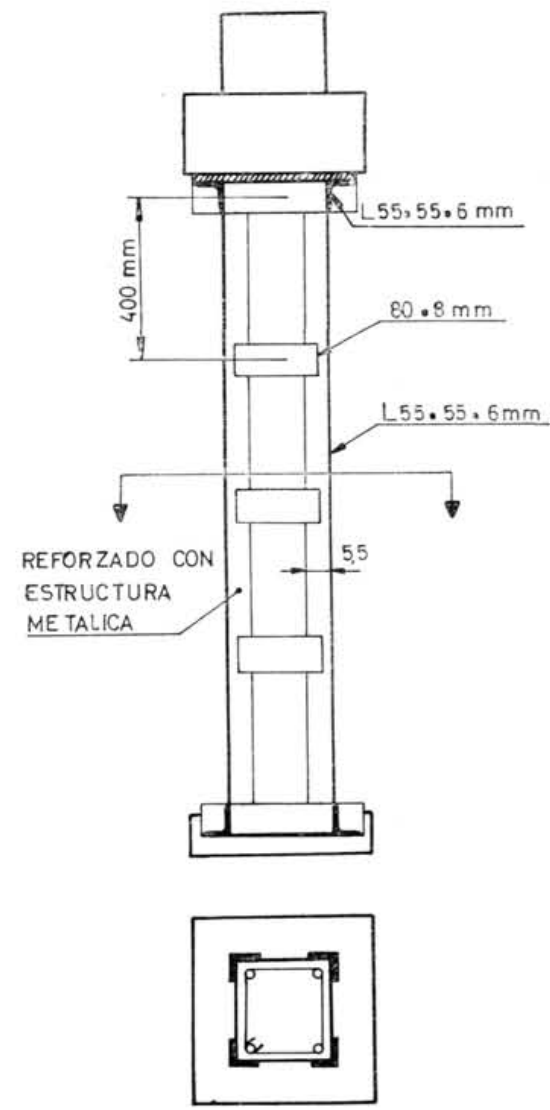

Los valores característicos previstos en el proyecto para la calidad del hormigón y la armadura de los pilares teóricos han sido, respectivamente, de 180 y $2.400 \mathrm{kp} / \mathrm{cm}^{2}$. La resistencia real obtenida ha sido para el hormigón de $110 \mathrm{kp} / \mathrm{cm}^{2}$ (60\% de la de proyecto), por lo que se ha procedido al refuerzo.

Este se ha realizado con el criterio de prescindir por completo del pilar original mal realizado, y que el refuerzo solo, a compresión simple, fuera capaz de soportar la carga de proyecto del pilar original.

No se ha pretendido que el refuerzo trabajara como zuncho del pilar original, aunque existe también esta posibilidad de refuerzo utilizando un sistema de estribos adecuado.

Deduzcamos la carga de proyecto del pilar original $(\mathbf{N})$ de acuerdo con los datos anteriores y la fórmula de la Instrucción Española EH-73.

$\gamma \mathbf{n} \cdot \gamma \mathbf{f} \cdot \mathbf{N}=0,85 \mathbf{b} \cdot \mathbf{h} \cdot 0,9 \cdot \frac{\mathbf{f c k}}{\gamma \mathbf{c}}+\mathbf{A}_{\mathrm{s}} \cdot \frac{\mathbf{f y k}}{\gamma_{\mathbf{s}}} ;$ siendo para este caso:

$$
\begin{aligned}
\gamma \mathbf{n} & =1,2 \\
\gamma \mathbf{f} & =1,6 \\
\mathbf{b}=\mathbf{h} & =25 \mathrm{~cm} \\
\mathbf{f c k} & =180 \mathrm{kp} / \mathrm{cm}^{2} \\
\gamma \mathbf{c} & =1,5 \\
\mathbf{A}_{\mathrm{s}} & =4 \times 0,78 \mathrm{~cm}^{2} \\
\mathbf{f y k} & =2.400 \mathrm{kp} / \mathrm{cm}^{2} \\
\gamma \mathbf{s} & =1,15
\end{aligned}
$$

Sustituyendo estos valores en la fórmula obtenemos una carga de proyecto de $33.275 \mathrm{kp}$.

El refuerzo en hormigón (fig. 1) ha consistido en aumentar la sección transversal del pilar original a $35 \times 35 \mathrm{~cm}$, mediante una capa exterior de hormigón de $180 \mathrm{kp} / \mathrm{cm}^{2}$ de $5 \mathrm{~cm}$ de gruesa, con lo que prácticamente se ha duplicado la sección primitiva de $25 \times 25 \mathrm{~cm}$. Se ha colocado en el hormigón añadido 4 armaduras lisas de $10 \mathrm{~mm}$ de diámetro y estribos lisos de $6 \mathrm{~mm}$ de diámetro cada $15 \mathrm{~cm}$. 
El refuerzo metálico (fig. 1) ha consistido en adosar a las cuatro aristas, angulares de $55 \times 55 \times 6 \mathrm{~mm}$, unidos mediante presillas de $80 \times 8 \mathrm{~mm}$, separadas $400 \mathrm{~mm}$. La carga capaz de ser aguantada por estos cuatro angulares es la siguiente:

$$
\mathbf{N}=\mathbf{A}_{\mathbf{s}} \cdot \frac{\mathbf{f y k}}{\gamma \mathbf{f} \cdot \gamma \mathbf{s}} ;
$$

en la que:

$$
\begin{aligned}
\gamma \mathbf{f} & =1,6 \\
\gamma \mathbf{s} & =1,1 \\
\mathbf{f y k} & =2.400 \mathrm{kp} / \mathrm{cm}^{2} \\
\mathbf{A}_{\mathrm{s}} & =4 \times 6,31 \mathrm{~cm}^{2}
\end{aligned}
$$

Sale para el valor de la carga $\mathbf{N}=34.400 \mathrm{kp}$, ligeramente superior a los $33.275 \mathrm{kp}$, carga de proyecto del pilar original.

Para poner de manifiesto el carácter aislado del refuerzo y su acción contra el forjado superior e inferior, se ha hecho en la parte superior del pilar un ensanchamiento de $45 \mathrm{~cm}$ de lado y $20 \mathrm{~cm}$ de grueso, que representaba la presencia del forjado. Se ha supuesto, por ser este el caso más desfavorable, la presencia de un forjado sin vigas salientes, con armadura principal en una sola dirección compuesta de cuatro redondos de acero corrugado de $20 \mathrm{~mm}$ de diámetro: tres en la parte superior y una en la inferior.

Atendiendo a la condición de simetría del refuerzo respecto al forjado superior e inferior se ha ensayado (fig. 1) una pieza que tiene $1,60 \mathrm{~m}$ de fuste de pilar, $20 \mathrm{~cm}$ de ensanchamiento representando el forjado, y $20 \mathrm{~cm}$ del tamaño primitivo del pilar que simboliza el pilar del piso superior. Es decir, se ha ensayado prácticamente la mitad del pilar, cogiendo la parte superior que es la que, por la posición de las armaduras del forjado, queda más débil desde el punto de vista de rotura de este último por empuje del refuerzo. (Más adelante haremos algunas observaciones sobre el forjado inferior.)

Aunque se han utilizado en el presente trabajo pilares de escuadría pequeña para hacer los ensayos de refuerzo, las conclusiones que se sacan de los ensayos pueden también ser útiles para el estudio de los problemas de refuerzo de pilares de mayor sección, teniendo en cuenta la circunstancia desfavorable de que el espesor de forjado no crece en proporción con la escuadría de los pilares.

\section{MATERIALES Y PROCESO DE ELABORACION DE LOS REFUERZOS}

El conjunto de los trabajos experimentales ha consistido en:

a) La preparación de 6 series de 3 pilares idénticos cada uno.

b) En el refuerzo con hormigón y con angulares de dos de los pilares de cada serie, dejando el tercero para comparación.

c) En el ensayo de todos los pilares a compresión simple, midiendo cargas, acortamientos y tensiones en armaduras y angulares mediante extensómetros óhmicos.

Los pilares originales, junto con el ensanchamiento del forjado, se confeccionaron con hormigón de árido calizo de machaqueo de $30 \mathrm{~mm}$ de tamaño máximo, dosificación de $200 \mathrm{~kg} / \mathrm{m}^{3}$ de cemento $\mathrm{P}-350$ y relación agua/ cemento de 1,23; todo con el objeto de obtener un "mal" hormigón de una resistencia media próxima a los $110 \mathrm{kp} / \mathrm{cm}^{2}$.

Posteriormente, para materializar la presencia del pilar del piso superior se hormigonó sobre la parte superior del forjado una cabeza de hormigón, de $25 \times 25 \mathrm{~cm}$ de sección y $20 \mathrm{~cm}$ de altura, para el que se utilizó una dosificación de $375 \mathrm{~kg} / \mathrm{m}^{3}$ de cemento y una relación agua/cemento de 0,7 . Finalmente, en la parte inferior del pilar se hizo una pequeña zapata de hormigón para mejorar el asiento sobre la prensa.

El primer tipo de refuerzo ha consistido en aumentar la sección con $5 \mathrm{~cm}$ de hormigón por cada lado, tal como se indica en la figura 1. La calidad del hormigón que se precisaba era $180 \mathrm{kp} / \mathrm{cm}^{2}$, con objeto de que el refuerzo fuera de resistencia análoga a la del pilar de proyecto.

Para conseguir esta resistencia se empleó un hormigón de $315 \mathrm{~kg} / \mathrm{m}^{3}$ de dosificación, con el mismo árido calizo, pero de $12 \mathrm{~mm}$ de tamaño máximo, y una relación agua/cemento de 0,88 , con el fin de obtener un hormigón plástico-fluido que se pudiera introducir y compactar bien en el espesor de $5 \mathrm{~cm}$. Con objeto de aproximarnos a las condiciones de la obra, este hormigón se vertió por unos orificios practicados en la zona que simula el forjado, con los pilares en posición vertical, vibrándose convenientemente y no apreciándose después coqueras o defectos de compactación. Se apiconó previamente el pilar 
primitivo para aumentar la adherencia entre el hormigón antiguo y nuevo.

Para controlar las calidades de los hormigones, tanto del pilar original como del refuer- zo y de la cabeza y pie, se confeccionaron probetas cilíndricas de $15 \times 30 \mathrm{~cm}$, que en la fecha de ensayo de los mismos pilares dieron los resultados que se presentan en el cuadro 1.

CUADRO 1

\begin{tabular}{|c|c|c|c|c|c|c|c|}
\hline \multirow{3}{*}{ Material } & \multicolumn{7}{|c|}{$\begin{array}{l}\text { RESISTENCIA A COMPRESION DEL HORMIGON * } \\
\qquad\left(\mathrm{kp} / \mathrm{cm}^{2}\right)\end{array}$} \\
\hline & \multicolumn{6}{|c|}{ Serie de pilares números: } & \multirow{2}{*}{$\begin{array}{l}\text { Valores } \\
\text { significativos }\end{array}$} \\
\hline & 1 & 2 & 3 & 4 & 5 & 6 & \\
\hline \multirow{6}{*}{ Pilar original } & 106,5 & 114,4 & 116,0 & 96,3 & 102,0 & 99,2 & \multirow{4}{*}{$\begin{array}{c}\text { Media general } \\
110,6\end{array}$} \\
\hline & 115,5 & 113,3 & 117,5 & 111,0 & 100,2 & 90,6 & \\
\hline & 126,0 & 122,3 & 114,5 & 116,5 & 107,8 & 99,7 & \\
\hline & 102,0 & 113,8 & 121,0 & 94,0 & 87,7 & 105,0 & \\
\hline & 124.5 & 131,4 & 143,0 & 112,0 & 82,2 & 121,2 & \multirow{2}{*}{$\begin{array}{l}\text { Coeficiente } \\
\text { de variación }\end{array}$} \\
\hline & 108,0 & 111,0 & 137,0 & 113,0 & 110,5 & 96,4 & \\
\hline Media ... & 113,7 & 117,7 & 124,8 & 107,1 & 98,4 & 102,0 & $12,0 \%$ \\
\hline \multirow{9}{*}{$\begin{array}{l}\text { Hormigón } \\
\text { del refuerzo }\end{array}$} & - & 181,2 & 171,0 & 192,0 & 165,4 & 135,0 & \multirow{7}{*}{ Media general } \\
\hline & 240,0 & 184,0 & 238,0 & 165,5 & 144,5 & 139,0 & \\
\hline & 249,0 & 197,6 & 192,0 & 164,5 & 157,5 & 137,2 & \\
\hline & 260,0 & 158,6 & 167,0 & 188,0 & 183,0 & 128,2 & \\
\hline & 238,0 & 167,6 & 164,5 & 162,5 & 168,0 & 123,6 & \\
\hline & 265,5 & 179,5 & 178,5 & 177,0 & 151,2 & 139,5 & \\
\hline & 246,5 & 244,6 & 198,0 & 189,0 & 170,5 & 125,2 & \\
\hline & - & 184,0 & 202,0 & 150,0 & 161,5 & 139,5 & \multirow{2}{*}{$\begin{array}{l}\text { Coeficiente } \\
\text { de variación }\end{array}$} \\
\hline & - & 159,1 & 205,0 & 143,5 & 181,2 & 136,5 & \\
\hline Media ... & 249,8 & 184,0 & 190,6 & 170,2 & 164,7 & 133,7 & $20.5 \%$ \\
\hline \multirow{2}{*}{$\begin{array}{l}\text { Cabezas } \\
\text { (para ensayo) }\end{array}$} & 352,5 & 342,6 & 324,0 & 300,0 & 304,2 & 276,3 & \multirow{2}{*}{$\begin{array}{l}\text { Media general } \\
\quad 325,0\end{array}$} \\
\hline & 404,0 & 297,3 & 353,0 & 309,0 & 347,0 & 296,0 & \\
\hline \multirow{2}{*}{$\begin{array}{c}\text { Zapatas } \\
\text { (para ensayo) }\end{array}$} & 195,5 & 238,4 & 217,0 & 157,5 & 200,5 & 228,0 & \multirow{2}{*}{$\begin{array}{l}\text { Media general } \\
\qquad 202,3\end{array}$} \\
\hline & 191,0 & 231,6 & 213,0 & 148,0 & 186,5 & 221,0 & \\
\hline
\end{tabular}

- Probetas correspondientes a pilar original conservadas en agua.

Probetas correspondientes al hormigón de refuerzo conservadas al aire.

Probetas correspondientes a cabezas y zapatas conservadas al aire.

Las armaduras y estribos utilizados en los pilares originales y refuerzos fueron de redondo liso en acero dulce corriente AE24L, de fyk $=2.400 \mathrm{kp} / \mathrm{cm}^{2}$. El corrugado de la zona del forjado fue estirado en frío del tipo AE42F, de $\mathbf{f y k}=4.200 \mathrm{kp} / \mathrm{cm}^{2}$.

El segundo tipo de refuerzo estudiado ha consistido en añadir a las cuatro esquinas del pilar otros tantos angulares de $55 \times 55 \times$ $\times 6 \mathrm{~mm}$ unidos entre sí mediante presillas de $80 \times 8 \mathrm{~mm}$ cada $400 \mathrm{~mm}$. Estas presillas se soldaban precalentadas para conseguir al enfriarse un apriete de los angulares al pilar.
En la parte inferior y superior del pilar, bajo el ensanchamiento que simula el forjado, se soldaron horizontalmente unos trozos del mismo angular para conseguir el apoyo de los angulares del fuste, tal como se indica en la figura 1. Con un mortero fino se rellenaron los pequeños espacios que quedan entre estos últimos trozos de angular y su base de apoyo, estando el pilar en posición vertical, para aproximarse lo más posible a las condiciones de la práctica. La calidad de estos angulares fue de A37b de la Norma MV 102, con un límite elástico mínimo de $2.400 \mathrm{kp} / \mathrm{cm}^{2}$. 


\section{RESULTADOS DE LOS ENSAYOS DE ROTURA}

Después de realizar los refuerzos quedaban para el ensayo seis series de pilares, formadas cada una por una unidad tal como se fabricó, otra reforzada con hormigón y la tercera reforzada con estructura metálica (foto número 1). Los tres pilares de cada serie se ensayaron en la misma fecha, utilizando una prensa de $250 \mathrm{Mp}$ de fuerza, capaz para pilares de $2,00 \mathrm{~m}$ de alto y $45 \times 45 \mathrm{~cm}$ de sección, provista de rótula.

La edad del hormigón en el momento de la rotura fue de 100 días para el del pilar primitivo y 50 días para el del refuerzo, para aproximarnos a las condiciones de obra.

Las resultados de los ensayos de compresión aparecen en el cuadro 2, en el que se incluyen las relaciones entre las resistencias de los pilares reforzados por los dos procedimientos, con la resistencia del pilar sin reforzar, permitiendo apreciar la mejora resistente que proporcionan.

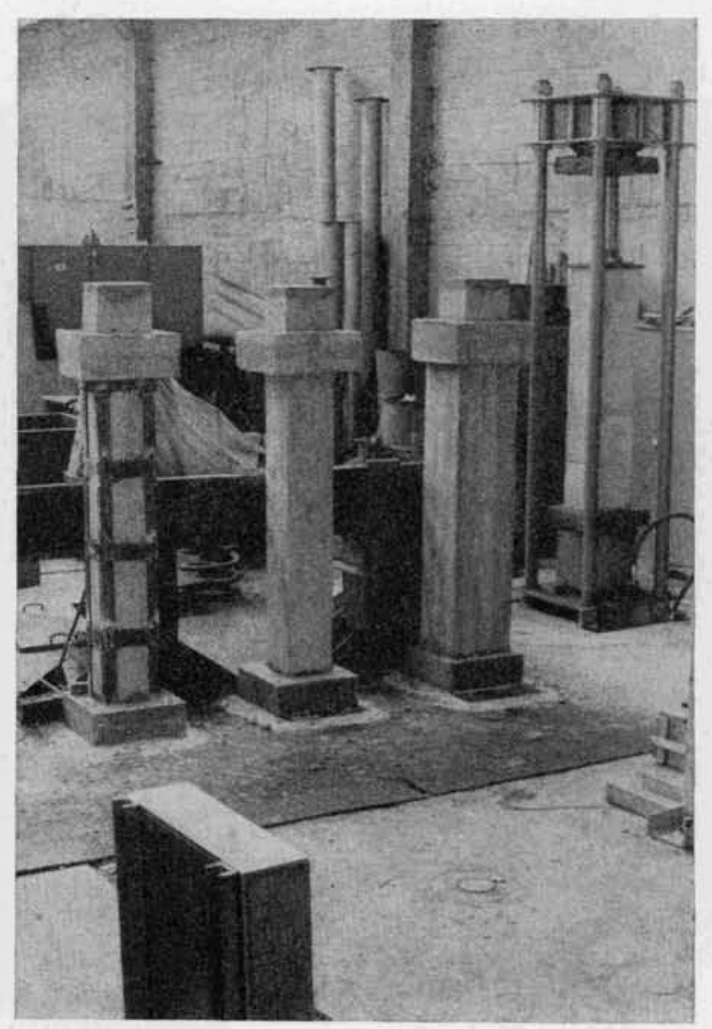

Foto 1

CUADRO 2

\begin{tabular}{|c|c|c|c|c|c|c|c|}
\hline \multirow{3}{*}{ Estado del pilar } & \multicolumn{7}{|c|}{$\begin{array}{l}\text { RESISTENCIA DE LOS PILARES SIN Y CON REFUERZO } \\
(\mathrm{kp})\end{array}$} \\
\hline & \multicolumn{6}{|c|}{ Serie de pilares números: } & \multirow{2}{*}{ Media } \\
\hline & 1 & 2 & 3 & 4 & 5 & 6 & \\
\hline & & & & & & 1 & \\
\hline Sin reforzar (1) & 86.044 & 87.418 & 87.143 & 78.071 & 74.223 & 80.270 & 82.195 \\
\hline $\begin{array}{c}\text { Refuerzo } \\
\text { en hormigón (II) }\end{array}$ & 158.650 & 147.250 & 132.670 & 132.670 & 144.070 & 132.050 & 141.227 \\
\hline $\begin{array}{c}\text { Refuerzo } \\
\text { metálico (III) }\end{array}$ & 145.350 & 132.050 & 124.450 & 105.450 & 126.975 & 106.720 & 123.499 \\
\hline Relación (II)/(I) & 1,84 & 1,68 & 1,52 & 1,70 & 1,94 & 1,64 & 1,72 \\
\hline Relación (III) /(I) & 1,70 & 1.51 & 1,43 & 1,35 & 1.71 & 1,33 & 1,50 \\
\hline
\end{tabular}

De las citadas relaciones se desprende en primer lugar la baja eficacia de los refuerzos aislados, tal como han sido proyectados, incluso para este caso de pequeña sección transversal del pilar. Sólo aumentan el $50 \%$ (metálico) o el $72 \%$ (hormigón) de la resistencia del pilar defectuoso, cuando estaban proyectados para suministrar el $100 \%$ de la resistencia del pilar de proyecto. El aumento de resistencia proporcionado por el refuerzo metálico coincide bastante bien con la resistencia al esfuerzo cortante del forjado. La mayor eficacia comprobada del refuerzo con hormigón será producida por el rozamiento y la adherencia entre los dos hormigones viejo y nuevo. 
Un dato de interés es la relación entre los resultados de la rotura de los pilares y la que teóricamente hubiera tenido el pilar correctamente hecho según el proyecto. Para poder confeccionar el cuadro correspondiente calculamos primero esta carga de rotura teórica, sin emplear el coeficiente de cansancio, debido a que los ensayos de rotura que hemos hecho pueden considerarse como ensayos rápidos:

$$
\mathbf{N}_{\mathrm{r}}=\mathbf{b} \cdot \mathbf{h} \cdot \mathbf{0 , 9} \cdot \mathbf{f c k}+\mathbf{A}_{\mathrm{s}} \cdot \mathbf{f y k} ;
$$

siendo:

$$
\begin{aligned}
\mathbf{b} & =\mathbf{h}=25 \mathrm{~cm} . \\
\mathbf{f c k} & =180 \mathrm{kp} / \mathrm{cm}^{2} . \\
\mathbf{A}_{\mathrm{s}} & =4 \times 0,78 \mathrm{~cm}^{2} . \\
\mathbf{f y k} & =2.400 \mathrm{kp} / \mathrm{cm}^{2} .
\end{aligned}
$$

Con lo que resulta:

$$
\mathbf{N}_{\mathrm{r}}=108.740 \mathrm{kp} \text {. }
$$

Los valores de las relaciones para todos los pilares ensayados figuran en el cuadro 3 .

\section{CUADRO 3}

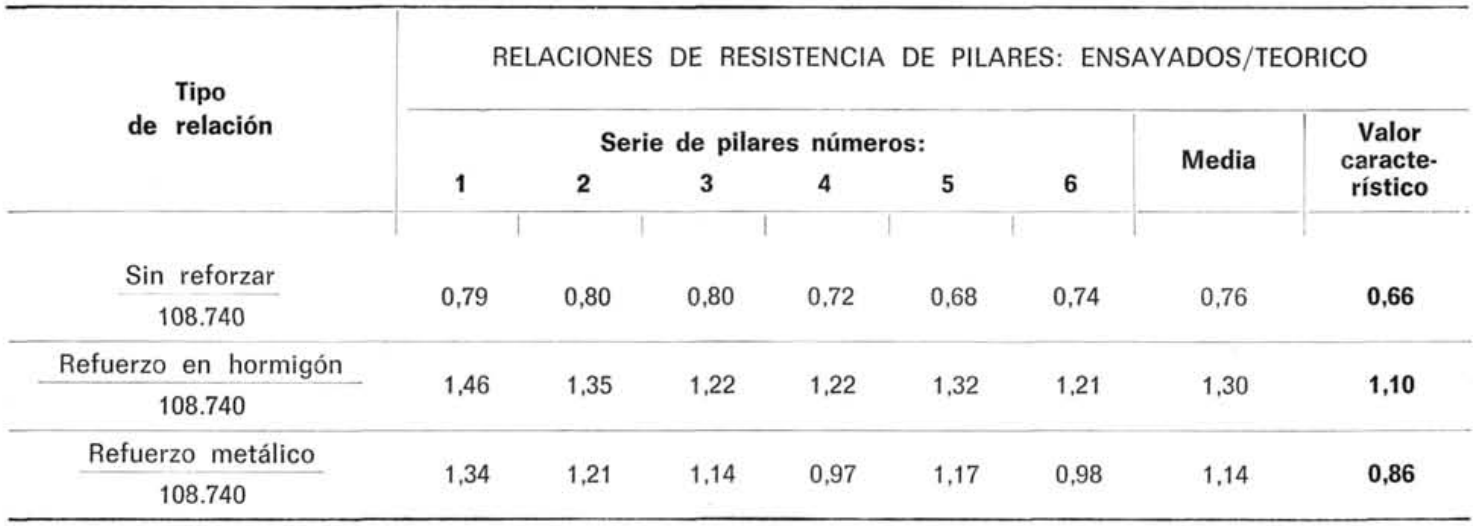

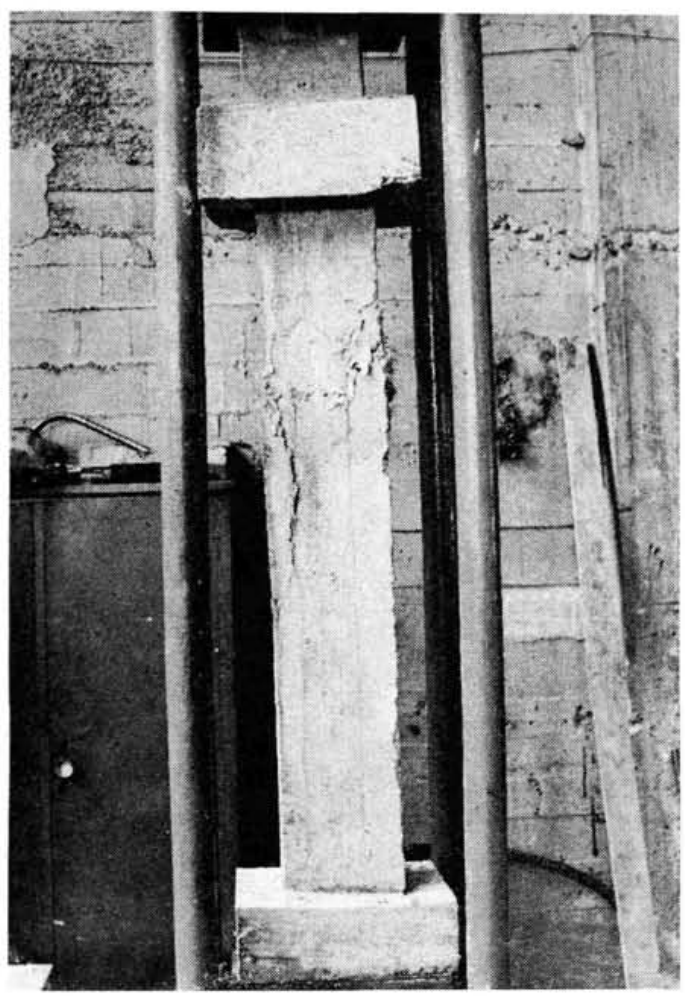

Foto 2
Refiriéndonos a dicho cuadro, se observa, para nuestros particulares tamaños pilar/forjado, que tanto con el refuerzo metálico como de hormigón se supera en valor medio en la rotura la resistencia máxima calculada para el pilar teórico. Sin embargo, resulta más eficaz el refuerzo de hormigón, tanto desde el punto de vista de nivel resistente como de regularidad de los resultados, puesto que el valor característico de las relaciones es en este caso de 1,10. Con el refuerzo metálico en dos casos no se ha superado la resistencia del pilar teórico y la dispersión es muy alta, lo que lleva a un valor característico de la relación de 0,86 y, por tanto, inseguro.

\section{FORMA DE LAS ROTURAS}

\section{a) Pilares sin reforzar}

En estos pilares las roturas se produjeron la mayor parte de las veces en la parte superior del fuste de los pilares, como parece lógico, ya que en esta zona el hormigón es algo más débil por segregación de la masa de hormigón y su menor compactación con respecto a la parte inferior. Este tipo de rotura puede verse en la foto número 2 . 


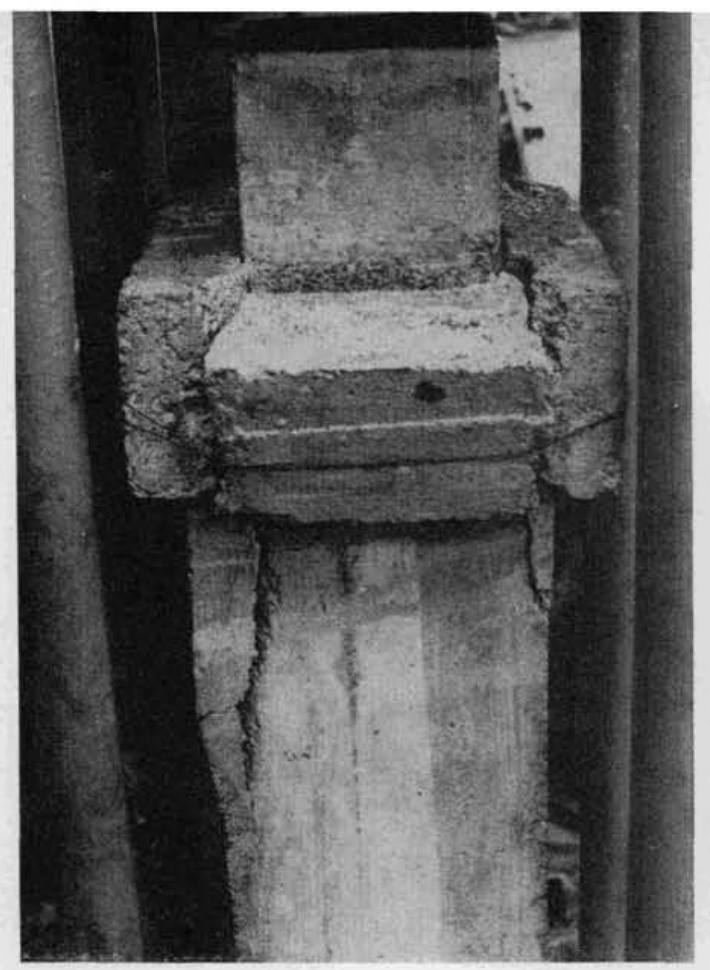

Foto 3

\section{b) Pilares reforzados con hormigón}

En este caso no se produjo la rotura del fuste reforzado, sino que en todos los casos la ruina se produjo por expansión del forjado original de baja calidad bajo la presión que le transmitía el dado del hormigón que representa el pilar superior y el empuje hacia arriba que le producía el refuerzo (fotos 3 y 4 y figura 2).

\section{c) Pilares reforzados con estructura metálica}

En este tipo de refuerzo las primeras fisuras aparecen en el fuste del pilar, en la misma zona que se produjeron las roturas de los pilares sin reforzar (foto 5). Después de esto la carga aplicada por la prensa seguía aumentando, produciéndose la ruina final por rotura del forjado, debido al punzonado hacia arriba empujado por el capitel de angulares contra el dado que simboliza el pilar superior. Es decir, se produce primero el fallo del pilar de hormigón y luego queda resistiendo por una mayor transmisión de esfuerzos a través de los angulares, hasta que falla el forjado por cortadura (fotos 5 y 6 y figura 2).

\section{DEFORMABILIDAD DEL PILAR ORIGINAL $Y$ DE LOS REFORZADOS}

En la figura 3 aparecen dibujadas a partir de valores medios las curvas carga-deformación de los tres tipos de pilares ensayados.

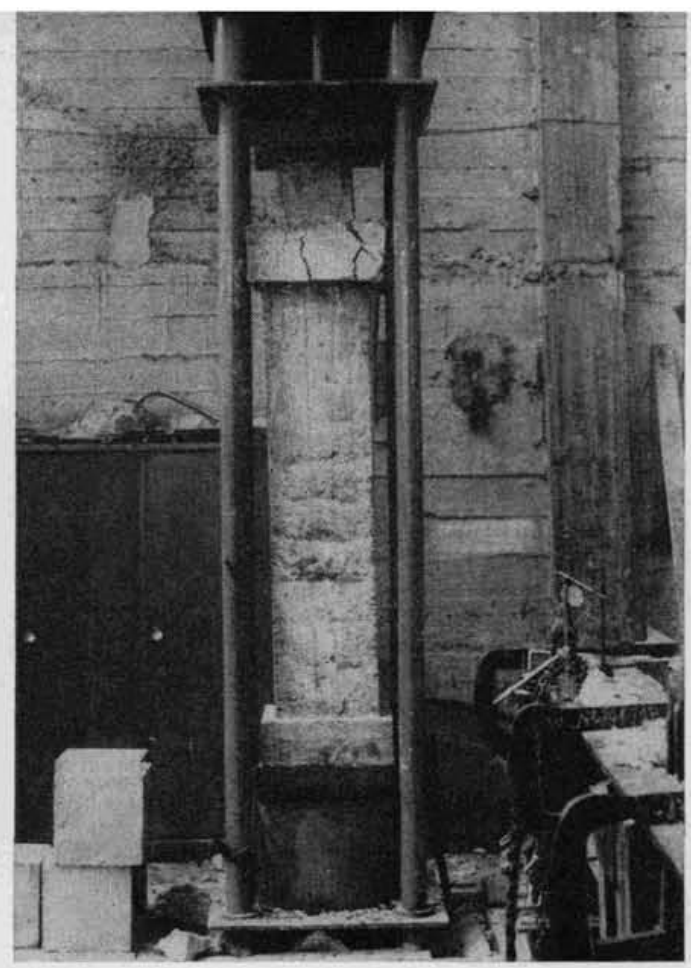

Foto 4

Se advierte en primer lugar la menor deformabilidad de los pilares reforzados respecto al de baja calidad sin reforzar y en particular el mejor comportamiento del reforzado con hormigón.
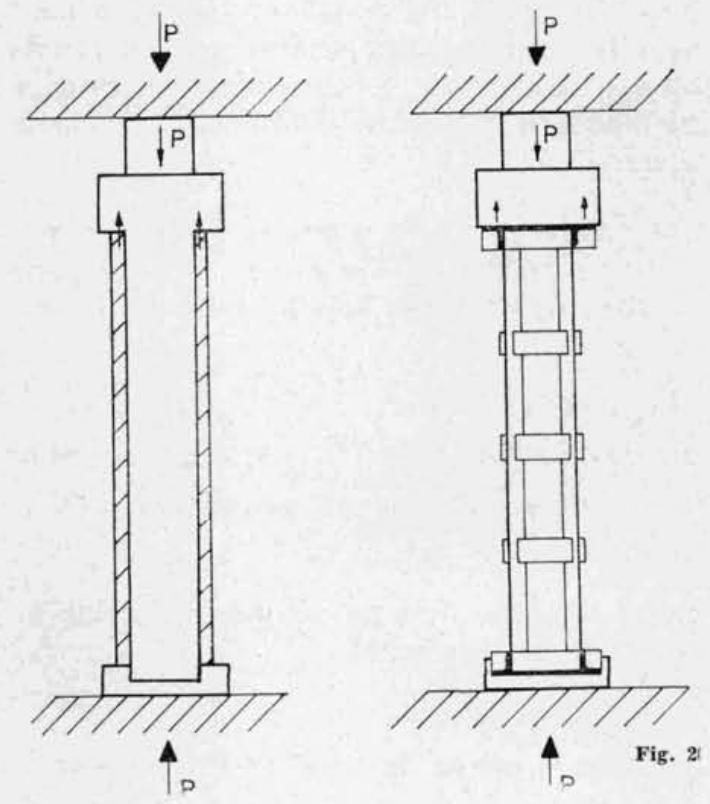

Para la carga de servicio (33.275 kp) la deformabilidad del reforzado en hormigón es el $80 \%$ de la correspondiente al refuerzo metálico. Son, pues, del mismo orden. Para niveles de la carga de rotura del pilar original $(108.740 \mathrm{kp}$ ) la deformabilidad del reforzado con perfiles es el $56 \%$ mayor del realizado en hormigón. 


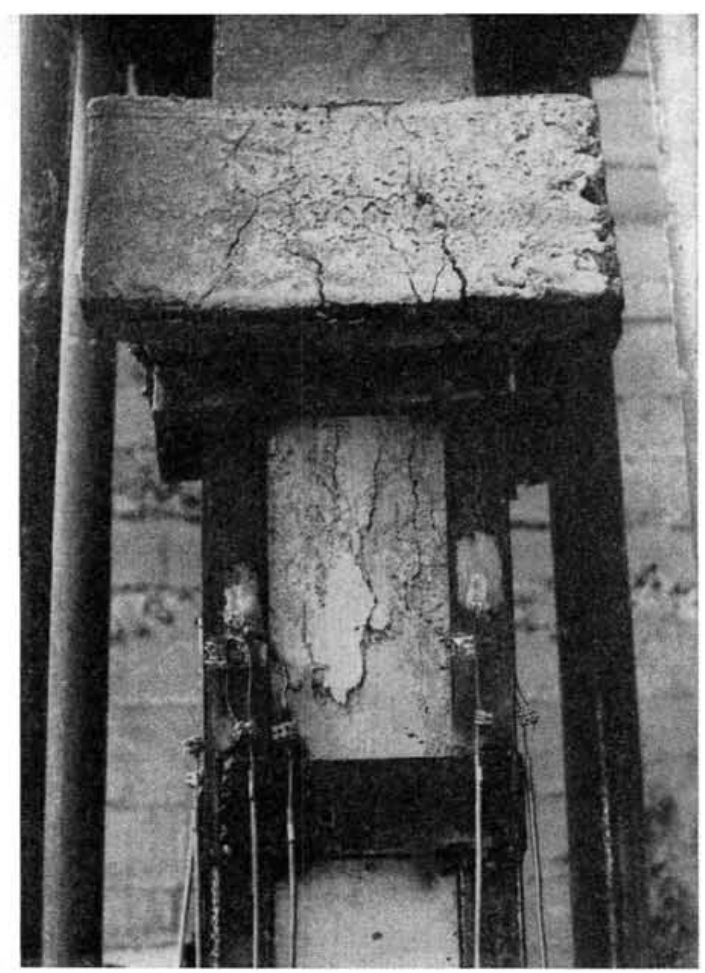

Foto 5

\section{REPARTO DE CARGAS ENTRE PILAR ORIGINAL Y REFUERZO}

Para poder realizar este estudio se pegaron extensómetros óhmicos en las armaduras originales de los pilares, en las del refuerzo de hormigón y en los angulares. Dicho pegado se hizo en diferentes aristas y a diferentes alturas a lo largo del fuste de los pilares, para poder obtener una información más completa.

Los valores medidos para las tensiones a lo largo del ensayo se han estudiado y comparado para tres niveles significativos de la carga:

a) la de servicio;

b) la de rotura del pilar teórico de proyecto;

c) la de rotura real de los pilares reforzados.

Como consecuencia se pueden hacer las siguientes observaciones:

\section{a) Para la carga de servicio (33.275 kp)}

1) En el caso de refuerzo con hormigón las armaduras de este refuerzo toman una deformación unitaria del orden del $15 \%$ superiores a las del pilar original. Esto significa, teniendo en cuenta el superior módulo de elasticidad del hormigón de la capa del refuerzo, que la carga que se transmite por este último es del orden del $40-45 \%$ superior a la que se trans-

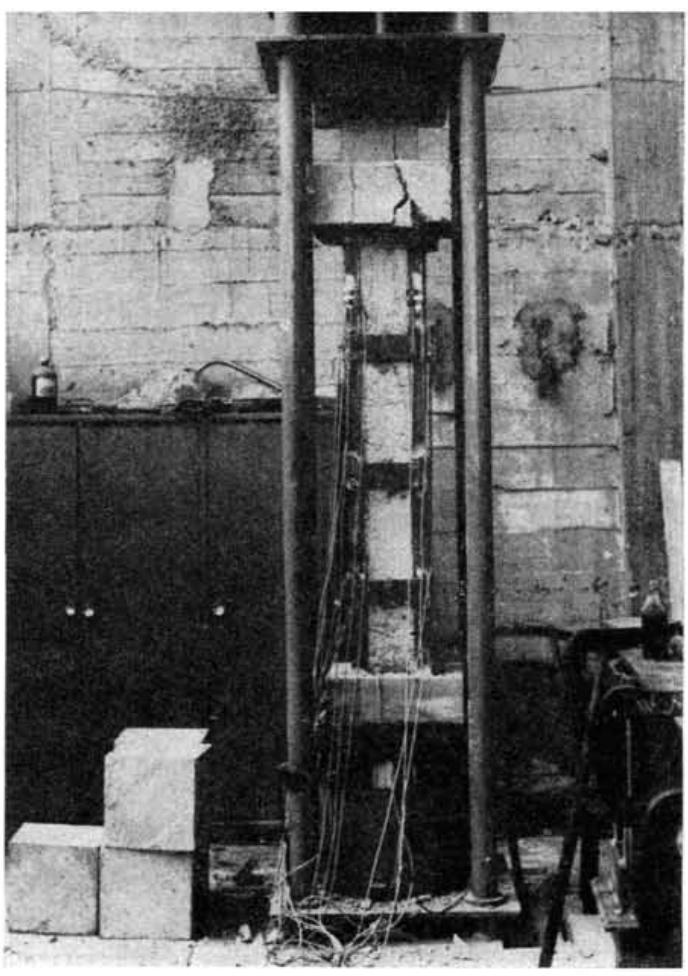

Foto 6

mite por la parte interior correspondiente al pilar original. De otra forma: para esta carga exterior el refuerzo absorbe el $60 \%$ y la parte interior de baja calidad el $40 \%$, aproximadamente.

2) Refiriéndonos al refuerzo con angulares, en la parte próxima inferior al forjado, éstos toman una deformación unitaria, que es, como valor medio, el $58 \%$ de la correspondiente a las armaduras del pilar original. Ello significa, al revés de lo sucedido con el refuerzo en hormigón, que al irse produciendo la carga el refuerzo de angulares va retrasado en la toma de carga respecto al pilar original de mala calidad, y de acuerdo con las medida, para la carga de servicio el refuerzo absorbe el $23 \%$ y la parte interior el $77 \%$ aproximadamente. Ello será debido a la dificultad de un buen acoplamiento entre los planos horizontales de los angulares de la base y capitel del refuerzo metálico y la superficie del forjado en la que apoyan (a pesar de haber sido aplicado mortero), y también a la flexión en ménsula de las mismas alas horizontales de los angulares.

En medidas realizadas más abajo, hacia la mitad del fuste, se ha visto un ligero aumento del trabajo de los angulares del refuerzo, como si hubiera una ligera transmisión del fuste de hormigón a los angulares por rozamiento, pero esta transmisión, además de pequeña, no creemos sea confiable en absoluto. 
b) Para la carga de rotura del pilar teórico (108.740 kp)

1) Para este valor del nivel de la carga se repiten prácticamente los valores dados para la carga de servicio en el caso de refuerzo en hormigón. La deformación unitaria de las armaduras de este refuerzo es un $17 \%$ superior a la de las armaduras del pilar original y se puede decir que para este nivel de la carga el refuerzo absorbe el $60 \%$ de la misma y la parte interior el $40 \%$ aproximadamente.

2) Para la carga de rotura del pilar teórico $(108.740 \mathrm{kp})$, en el caso de refuerzo metálico, los angulares siguen tomando menor deformación unitaria que la de las armaduras del pilar primitivo. Es la mitad de la de estas últimas. De acuerdo con las medidas para este nivel de carga el refuerzo absorbe el $32 \%$ de la carga y el pilar original el $68 \%$ aproximadamente.

c) Para la carga de rotura real de los pilares reforzados

1) En el caso del refuerzo en hormigón la carga de rotura media es de $141.277 \mathrm{kp}$, y puede decirse que en este momento el $42 \%$ lo resiste el refuerzo y el $58 \%$ el pilar original.

2) La rotura real de los pilares con refuerzo metálico sucede para un valor medio de $123.499 \mathrm{kp}$ y se puede calcular que el reparto de esfuerzos en este momento es de $34 \%$ para el refuerzo metálico y el $66 \%$ para el pilar original.

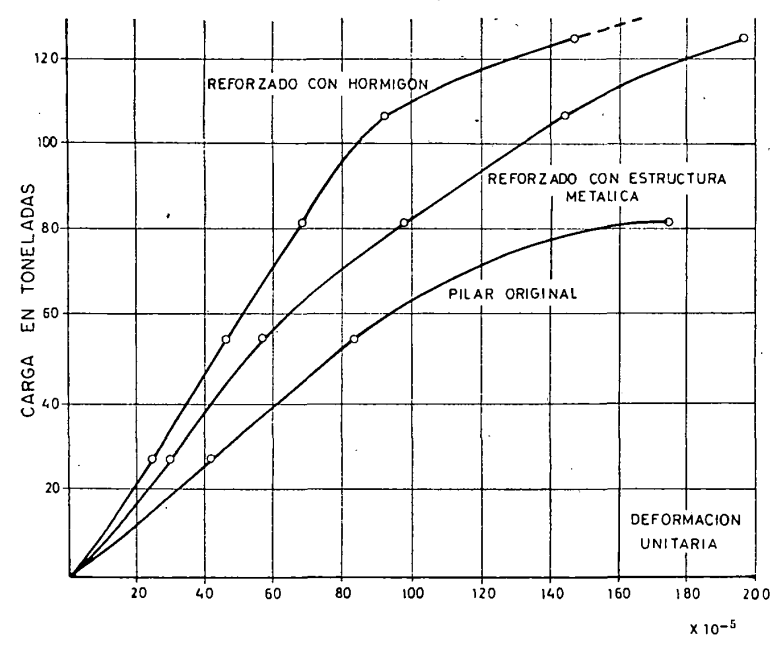

Fig. 3
Todo lo que se ha expuesto hasta ahora hay que tener en cuenta que ha sido basándose en ensayos rápidos, que no permiten poner en juego la diferencia en el comportamiento plástico diferido relativo del hormigón de mala calidad primitivo y cualquier tipo de refuerzo.

Pensamos que al ceder plásticamente el hormigón interior al refuerzo, se pondrá más en juego la cortadura del forjado que tiene un valor pequeño y que las relaciones resistencia pilares reforzados/resistencia pilar original, bajo cargas permanentes, serán menores de las obtenidas en los ensayos rápidos reflejados en el cuadro número 3.

\section{CONCLUSIONES}

En primer lugar, queremos manifestar que las observaciones que se hacen a continuación son función de los resultados obtenidos en la experimentación sobre los dos tipos particulares de refuerzo utilizados, que son frecuentes de encontrar en la práctica. Reconocemos, por otra parte, que hay otros métodos más elaborados de refuerzo que darán mejor resultado.

Una importante segunda observación, es que vamos a suponer que la sección de la columna del piso inmediatamente inferior es mayor que la del pilar que se refuerza, de tal forma que sólo sea crítica la cortadura del forjado superior.

Establecidas estas dos bases previas y contemplando los resultados podemos decir que estos refuerzos de pilares de baja calidad en pisos aislados, sea en hormigón o en estructura metálica, realizados de acuerdo con los dos métodos mencionados, resultan poco eficaces respecto a la resistencia intrínseca que aportan debido al fallo por aplastamiento o cortadura del forjado también de baja calidad.

Es, sin embargo, más eficaz, para la misma capacidad resistente de refuerzo aportada, el realizado en hormigón armado por su mayor carga de rotura, debida a la adherencia y rozamiento y por la regularidad de resultados.

Con refuerzo metálico, incluso comparando valores correspondientes a ensayos rápidos, se ha obtenido, para nuestras dimensiones, resistencia inferior a la carga de rotura del pilar teórico, hablando en términos de valores característicos. Bajo cargas permanentes el resultado sería aún más desfavorable. 
Con refuerzo de hormigón el asunto se ha presentado en forma más favorable, pues la relación característica de cocientes de resistencia es superior a la unidad. Esta relación bajará, creemos, cuando se trate de cargas permanentes.

Desde el punto de vista práctico se comprueba experimentalmente que el refuerzo metálico aislado se apoya totalmente en la resistencia al corte del forjado, y que por ello, hasta para la sección pequeña de pilar ensayada, no hay seguridad de elevación de la carga de rotura por encima de la resistencia proyectada para el pilar teórico. Por ello, y sobre todo para secciones mayores de pilar, a la hora de dimensionar un posible refuerzo metálico habrá que estudiar cuidadosamente la resistencia al corte de los forjados, extendiendo el refuerzo hacia arriba y hacia abajo lo que fuese necesario. Pero para confiar en este tipo de refuerzo es además necesario realizar un cuidadoso y eficaz ajuste entre el acero y el hormigón, y dar gran rigidez a los capiteles y bases metálicas, para evitar, como hemos vistos en nuestras experiencias, el retraso en la toma de carga del acero respecto al hormigón.

En el refuerzo con hormigón la mejora de resistencia no pone tanto en juego la resistencia al corte del forjado, debido a la adherencia y rozamiento entre hormigón antiguo y de refuerzo. De todas formas, para secciones de pilares mayores de las utilizadas en este trabajo creemos necesario hacer un estudio de las posibilidades de rotura de la cabeza por corte y aplastamiento, para, en su caso, mejorar la transmisión de esfuerzos en esa zona, mejorar la adherencia mediante lla- ves, taladros, etc., o, finalmente, reforzar algún piso superior e inferior.

Puede parecer sorprendente hablar de extender el refuerzo a algún piso inferior cuando hemos establecido como condición que éstos presenten mayor sección que el que se refuerza con lo que se produce apoyo del refuerzo, pero lo decimos para evitar transferencias de esfuerzos a una zona generalmente pequeña de sobreespesor.

En los forjados con vigas normales es mayor la resistencia al corte del forjado y, por tanto, las posibilidades del tipo de refuerzos aqui estudiados.

Finalmente, queremos hacer alguna consideración para el caso de que el pilar del piso inferior sea del mismo tamaño que el corres. pondiente al piso que se refuerza.

En este caso el empuje de corte por el refuerzo actúa en el mismo sentido al corte causado por la carga en el forjado del piso que se refuerza. No se suman totalmente debido a que el corte producido por el refuerzo es muy próximo a las paredes del pilar, no tomándose para el cálculo de corte en las normas las cargas comprendidas entre las caras del pilar y una línea separada la mitad del canto útil de la placa que, en este. caso, sería de $9 \mathrm{~cm}$.

Sin embargo, no parece oportuno confiar para apoyo del refuerzo en el corte del forjado, por lo que siempre sería necesario el bajar el refuerzo hasta el primer pilar que presente exceso de sección, pudiendo entonces aplicarse lo que se dijo anteriormente.

\section{résumé}

Efficacité résistante des poteaux en béton armé à faible qualité ren forcés pa dèux procédés différents J. L. Ramírez Ortiz,

M. Bárcena Díaz, ingénieur industriel

Dans cette recherche, on a voulu étudier la possibilité de renforcer les poteaux en béton arme, d'une basse qualité, dans, de étages isoles, en utilisant deux methodes du béton et la pose de la section ave acier laminé dans les quatre arêtes du poteau.

Le. travail de recherche a consisté en la fabrication, renforcement et essai de dix huit poteaux en béton armé de basse qua lité. A une certaine hauteur, on a dispos un élargissement pour. représenter la présence d'une dalle.

Des essais de compression on a obten des résultats et des conclusions sur l'efficacité des deux types de renforcement, sur la distribution de l'effort entre la section originale et celle du renforcement, sur le deformations et sur la configur

\section{summary}

Resistance of low quality reinforced concrete columns strengthened by two different methods

J. L. Ramirez Ortiz,

J. M. Barcena Diaz, industrial eng.

This research deals with the possibility of the strengthening of bad quality reinforce concrete columns, in isolated stories, by cross section with concrete, and the backing up of rolled steel angles to the edges of the column.

The experimental work has consisted in the manufacturing, strengthening and testing of eighteen reinforced concrete columns with low quality concrete. At a certain height above their bases a widening has been provided to represent the presence of a

From the compression tests data and conclusions concerning the strength efficiency of the two types of strengthening have been

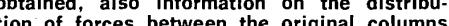
and of forces between the original columns deformations, cracking and type of failure.

\section{zusammenfassung}

Festigkeit von durch zwei verschiedene Verfahren verstärkte Stahbe: tonpfeilern geringer Qualität

J. L. Ramirez Ortiz, Prof. Dr. Ind. Ing. J. M. Bárcena Díaz, Ind. Ing

Die Untersuchung behandelt die Möglichkeit von Verstärkung in isolierten Wohnungen von Stahlbetonsäulen von geringer Qualität durch zwei gewöhnliche Methoden: Erwejterung des Querschnitts mit Beton und Stützen mit Winkel Stahlen von den Pfeilerkanten.

Die Versuche bestanden aus Herstellung, Verstärkung und Prüfung von 18 Stahlbetonerstarkung und Prufung von 18 Stahlbetonbracht worden, um die Anwesenheit einer flachen Platte darzustellen.

Von den Druckversuchen sind Daten und Schlussfolgerungen gezogen worden, in Bezug auf die Wirksamkeit der zwei Methoden, Kraftverteilung zwischen dem ursprung. lichen und der Verstärkung, Verformungen, Rissbildungen und Bruchform. 